\title{
Perspective
}

PERSPECTIVE Actualité en histoire de l'art

$1 \mid 2020$

Japon

\section{Entretien avec Takesada Matsutani}

par Valérie Douniaux

Valérie Douniaux et Takesada Matsutani

\section{CpenEdition}

Journals

Édition électronique

URL : http://journals.openedition.org/perspective/17938

DOI : 10.4000/perspective. 17938

ISSN : 2269-7721

Éditeur

Institut national d'histoire de l'art

\section{Édition imprimée}

Date de publication : 5 juin 2020

Pagination : 111-124

ISBN : 978-2-917902-89-9

ISSN : $1777-7852$

Référence électronique

Valérie Douniaux et Takesada Matsutani, «Entretien avec Takesada Matsutani », Perspective [En ligne], 1 | 2020, mis en ligne le 30 décembre 2020, consulté le 25 janvier 2021. URL : http://

journals.openedition.org/perspective/17938; DOI : https://doi.org/10.4000/perspective.17938 


\section{Entretien avec Takesada Matsutani}

par Valérie Douniaux

Takesada Matsutani est né en 1937 à Ōsaka, au Japon. Installé en France depuis 1966, il a mené une riche carrière internationale et a eu l'honneur en 2019, au Centre Pompidou, d'une exposition personnelle (fig. 1 ) qui présentait notamment une donation de 22 œuvres au musée. Matsutani a également fait don à l'Institut national d'histoire de l'art, en 2020, d'un ensemble exceptionnel de 92 estampes et livres d'artiste offrant un panorama complet de son travail dans le domaine de l'image imprimée.

De 1963 à 1972, Matsutani a été membre du groupe d'avant-garde Gutai (littéralement " concret "), fondé en 1954 par Yoshihara Jirō (1905-1972). Gutai prônait une relation directe de l'artiste à la matière et, en

1. Vue de l'exposition Takesada Matsutani au Centre Pompidou (26 juin 2019 -

23 septembre 2019), avec Circle-Green-2, Circle-Yellow-19, Circle-3-Blue, 2019, colle vinylique, acrylique sur toile montée sur contreplaqué, diamètre : $162 \mathrm{~cm}$, archives Matsutani.

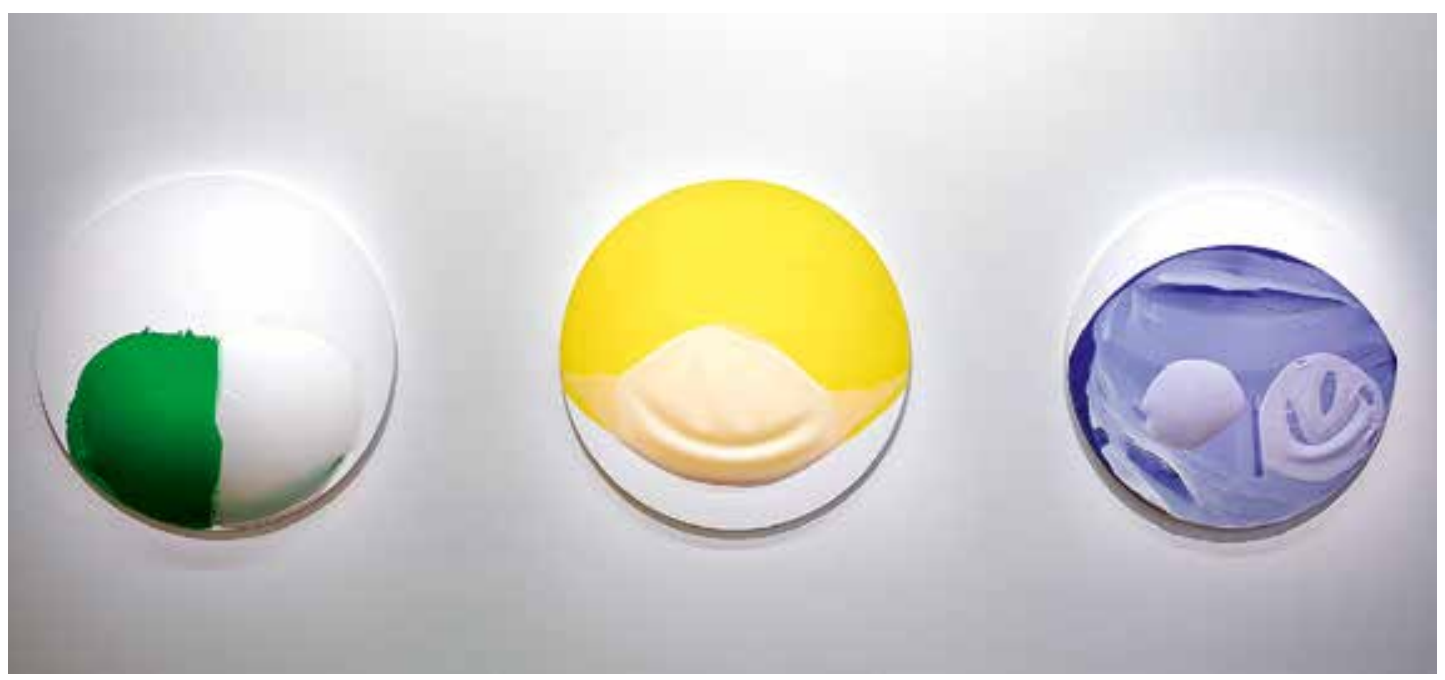




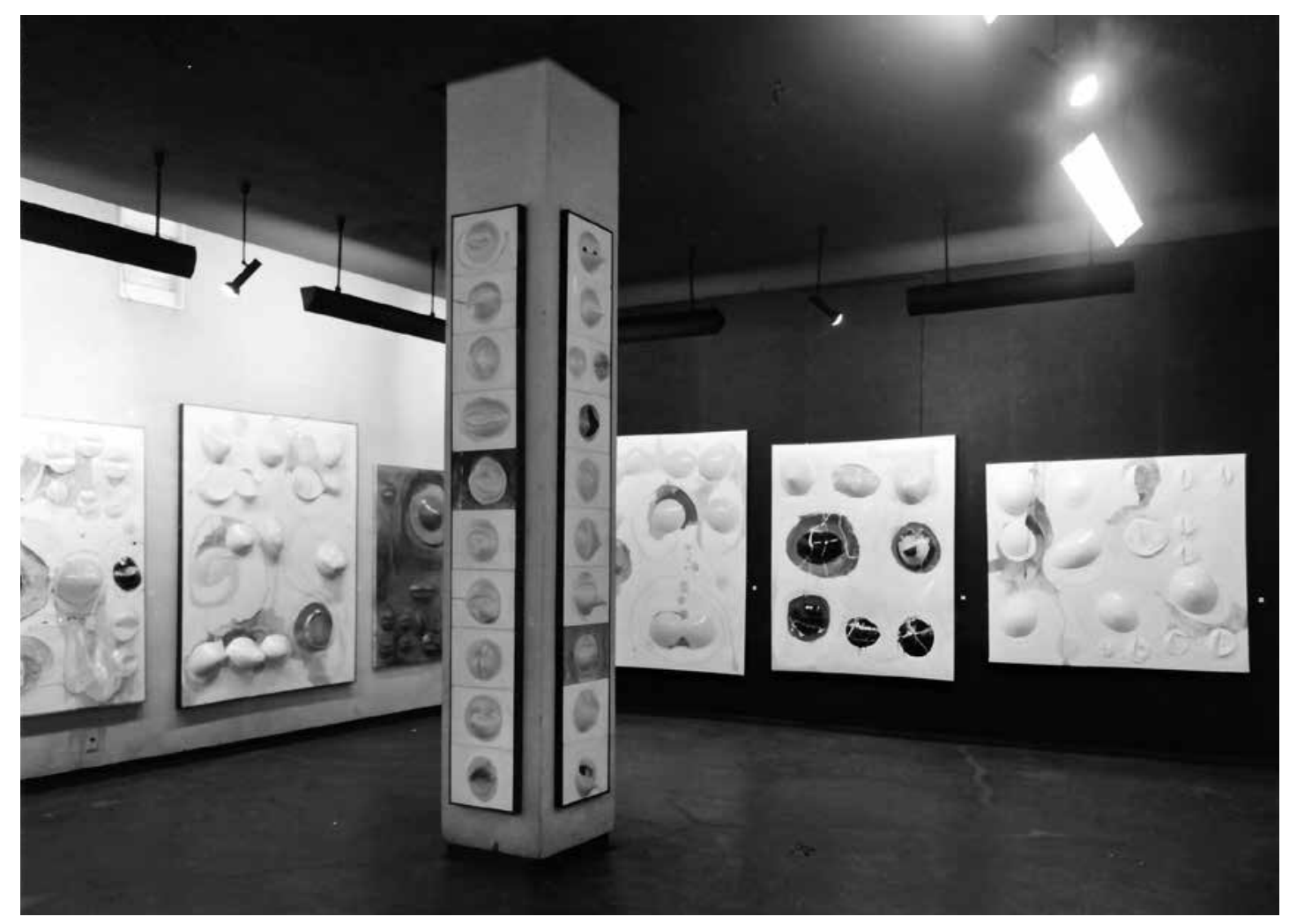

2. Vue de la première exposition personnelle de Matsutani à Ōsaka, à la Pinacothèque Gutai, 1963, archives Matsutani. véritable précurseur, concevait des installations et performances spectaculaires, menées dans des lieux inattendus (fig. 2). Matsutani a alors choisi comme outil de prédilection la colle à bois (colle vinylique), matériau moderne et bon marché, qu'il utilise encore aujourd'hui, faisant naître sur la toile ou le papier des volumes d'une grande sensualité. À partir du milieu des années 1970, les surfaces blanches et les volumes de colle sont recouverts de superpositions de traits de crayon graphite, d'un noir profond, aux reflets métalliques changeant selon les jeux de l'ombre et de la lumière. Les formats se font de plus en plus imposants au fil du temps, jusqu'aux installations spectaculaires présentées en 2017 à la Biennale de Venise (Stream Venice, fig. 3) et en 2019 au Centre Pompidou (Stream Pompidou). Si ces deux pans du travail de Matsutani, la créativité bouillonnante de Gutai et " l'œuvre au noir ", sont bien connus du public et ont fait l'objet de nombreuses expositions et publications, les années consacrées par Matsutani à la gravure, en particulier entre 1967 et 1972, et la brève période, plus ou moins contemporaine, durant laquelle il a adopté une abstraction proche du Hard-Edge américain, ont été beaucoup moins explorées et exposées. Une exposition d'œuvres Hard-edge, pour la plupart inédites, chez Hauser $\delta$ Wirth à Zürich en 2016, suivie d'une présentation de gravures, de plaques et de carnets de l'artiste chez Hauser \& Wirth Somerset en 2018, ont cependant permis de donner un coup de projecteur sur ces moments également importants du parcours de Matsutani,

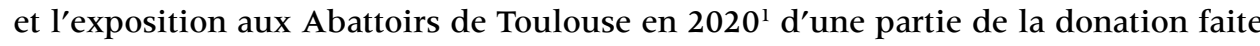
à l'INHA permettra de les mettre encore plus largement en avant.

[Valérie Douniaux] 


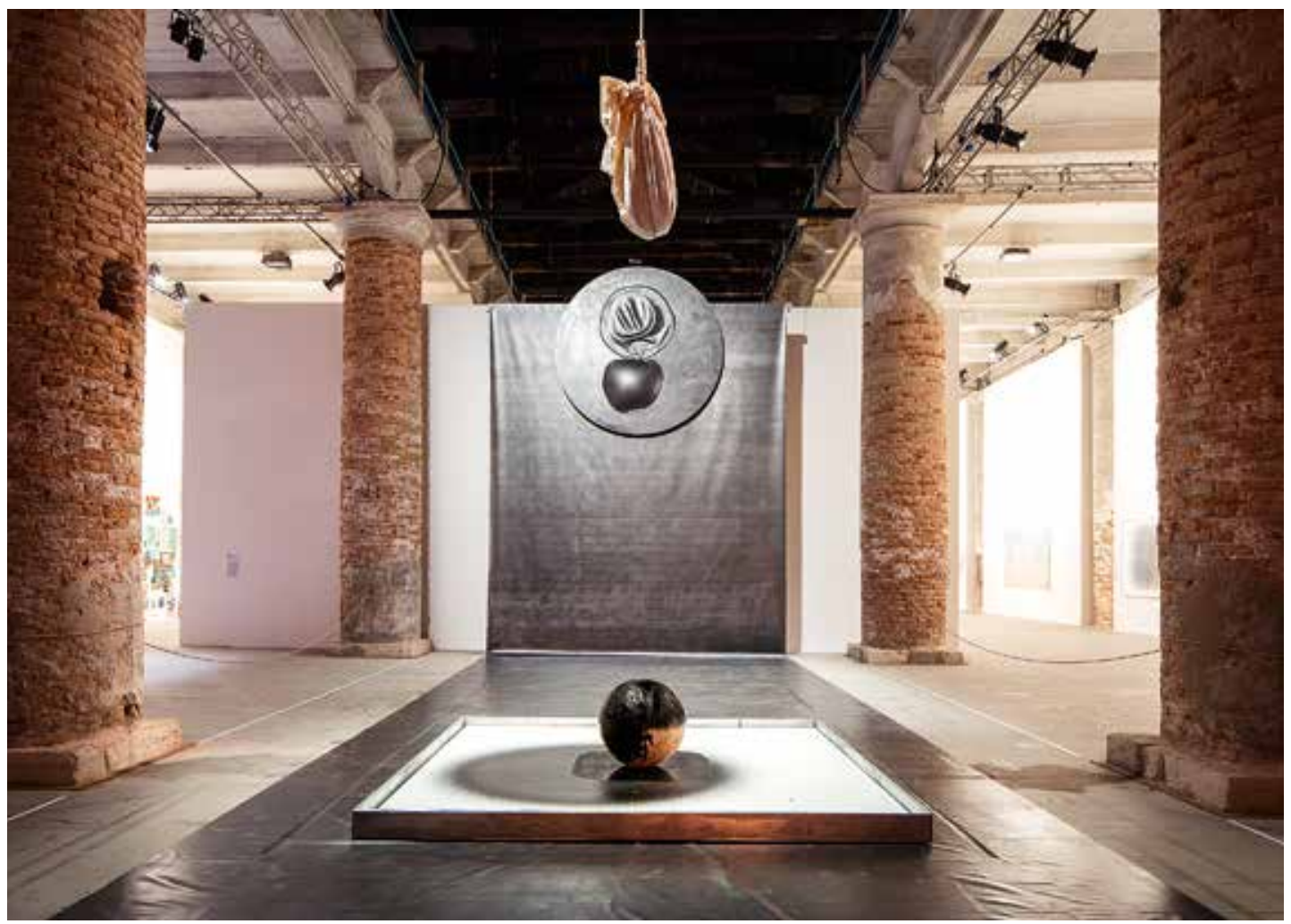

- Valérie Douniaux. Vous êtes arrivé en France en 1966, après plusieurs années de création intense au sein 3. Takesada Matsutani, Stream-Venice, 2017 crayon graphite, colle vinylique sur toile, crayon graphite sur toile, sac de toile avec encre sumi, corde, balle en bois, toile et bassin de Gutai. Vous n'aviez jamais quitté le Japon, le choc culturel a donc dû être assez important. Comment avez-vous vécu ces premiers mois loin de chez vous et ce bouleversement de votre environnement créatif? en zinc, environ $1600 \times 411 \mathrm{~cm}$, Biennale de Venise 2017, Viva Arte Viva, La Corderie, Pavilion of Colours and Mystical Joy.

- Takesada Matsutani. J'ai en effet ressenti un véritable choc culturel à mon arrivée en France. Tout me semblait différent du Japon. Ça m’a poussé à remettre en question ma place dans le monde, et mon mode d'expression. Aussi, quand un compatriote de ma classe de français m’a proposé de partir en voyage aux sources de la culture occidentale, de l'Égypte à l'Italie, j'ai immédiatement accepté. En plus, sur le plan pratique, je n'avais pas à Paris d'atelier me permettant d'utiliser la colle vinylique, je ne pouvais pas poursuivre la même ligne qu'au Japon. Je pense que je sentais aussi que j'étais arrivé au bout des possibilités de ce que je pouvais faire avec Gutai, je devais m'obliger à passer à autre chose. Je me suis souvenu des expositions de Stanley William Hayter (1901-1988) que j'avais pu voir au Japon et, en janvier 1967, j'ai décidé de pousser la porte de l'Atelier 17, présenté à Hayter par un compatriote, l'artiste Yayanagi Tsuyoshi (plus connu sous le nom de Yayanagi Go [1933-]). Hayter a accepté que j'intègre l'atelier et je me suis dès lors jeté dans l'apprentissage des techniques de la gravure (fig. 4).

- Valérie Douniaux. Vous avez débuté la gravure une fois en France, mais vous y intéressiezvous déjà au Japon? 


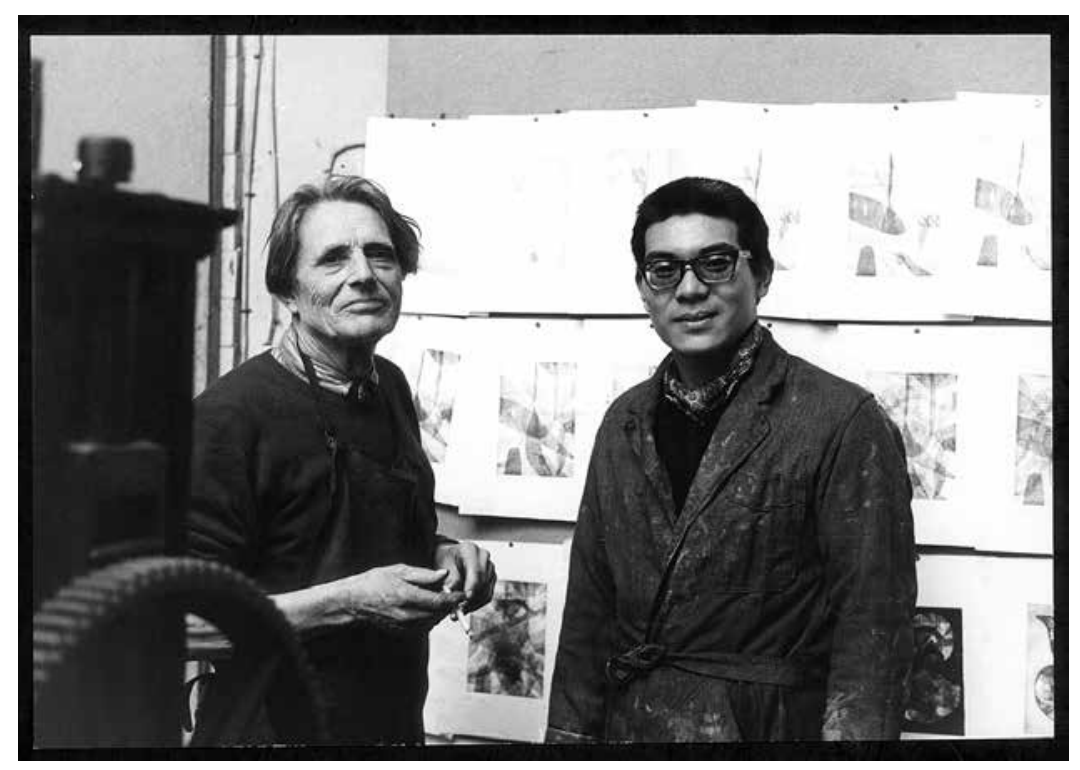

4. Matsutani avec Stanley William Hayter à l'Atelier 17.
- Takesada Matsutani. J'ai toujours souhaité faire de la gravure sur métal car, si le Japon a une grande tradition de gravure sur bois, les autres techniques y sont moins pratiquées. II y avait probablement des ateliers, mais je ne les connaissais pas, et les artistes de Gutai ne pratiquaient pas la gravure. Donc, à part quelques linogravures à l'école quand j'étais adolescent ou quelques essais personnels de monotypes au rouleau, je n'avais pas eu l'opportunité d'expérimenter les divers procédés de gravure. J'avais pu voir des reproductions d'œuvres occidentales dans la revue Bijutsu Techō [Cahiers d'art]. Surtout, j'avais découvert le travail de Hayter à la Tōkyō Print Biennale, où il avait remporté le Grand Prix en 1960, puis à Ōsaka, où il avait été invité à exposer suite à l'obtention de ce prix. Le monde de la gravure était alors en effervescence, avec de nombreuses expositions internationales, d'importantes biennales, qui offraient aux artistes de belles opportunités d'exposer leurs œuvres à l'étranger... J'ai moi-même envoyé ma candidature à de nombreuses biennales lorsque j'ai commencé à travailler à l'Atelier 17, et j'ai ainsi eu la chance de pouvoir présenter régulièrement mon travail et même de remporter des prix. J'ai pu aussi envoyer mes gravures au Japon, où je continuais à participer à toutes les expositions Gutai, notamment lors de l'exposition universelle d'Ōsaka en 1970.

- Valérie Douniaux. Pouvez-vous nous parler plus en détail de vos premiers essais dans le domaine de la gravure?

- Takesada Matsutani. Si I'on prend une œuvre comme Propagation T-50 (1967, fig. 5), qui est une des toutes premières, on retrouve très clairement les formes qui m'occupaient déjà au Japon. Ces motifs sont fortement teintés d'érotisme, mais en fait, à l'origine, si j'ai tranché ainsi dans mes volumes arrondis, c'était pour permettre à la colle de sécher plus rapidement. Le résultat, puissant et sensuel, m'a paru très intéressant, j'ai eu d'emblée la sensation d'une véritable conversation avec mon matériau, et j'ai donc poursuivi dans cette voie. Quand je suis arrivé en France, je ne pouvais plus travailler en volume faute de place, mais j'ai essayé de reproduire sur le papier les formes créées par la colle. J'étais passionné par cette possibilité d'exprimer les trois dimensions dans la planéité, qui s'accompagnait pour 
moi d'une découverte plus approfondie de la perspective linéaire occidentale. On ressent très vite dans mes gravures une volonté d'associer les deux, le volume, repensé dans l'espace plan, et la perspective (fig. 6).

- Valérie Douniaux. Votre intérêt se portait donc plus sur la forme et la perspective que sur la couleur, malgré la prédilection de Hayter pour celle-ci et la technique révolutionnaire qu'il avait élaborée, avec I'application de la couleur en trois passages?

- Takesada Matsutani. L'action painting dominait à l'Atelier 17 mais personnellement je trouvais cela déjà dépassé, je voulais autre chose (en plus je ne m'intéresse pas vraiment à la chimie de la gravure). L'art cinétique et l'art optique étaient très présents à Paris, j'allais souvent à la Galerie Denise René, je trouvais cela intéressant, en particulier le travail de Soto, qui avait fait une grande installation pour le musée d'Art moderne de la Ville de Paris. Je regardais beaucoup les autres artistes, je visitais les musées, en France et en Europe, je suis même allé voir, en Hollande, les œuvres de Mondrian et de De Stijl. Je découvrais une perspective très différente de celle que je connaissais au Japon.

Par ailleurs, j'ai retrouvé dans la gravure la puissance du noir et blanc que j'avais déjà ressentie en apprenant la calligraphie à l'école. J'ai toujours aimé aussi Odilon Redon, Hasegawa Kiyoshi (1891-1980)... ces artistes qui faisaient naître une atmosphère mystérieuse par le biais du noir et blanc. J'avais envie d'explorer tout cela, sans perdre de vue la dimension organique de mes œuvres. Et puis on associe souvent Hayter à la couleur mais c'était quelqu'un qui avait également une approche scientifique des choses. Il avait une formation d'ingénieur, de grandes connaissances en géométrie...

- Valérie Douniaux. Cette exploration de la perspective géométrique est flagrante dans des œuvres comme À l'avenir, L'Éternité ou L'angle vert.
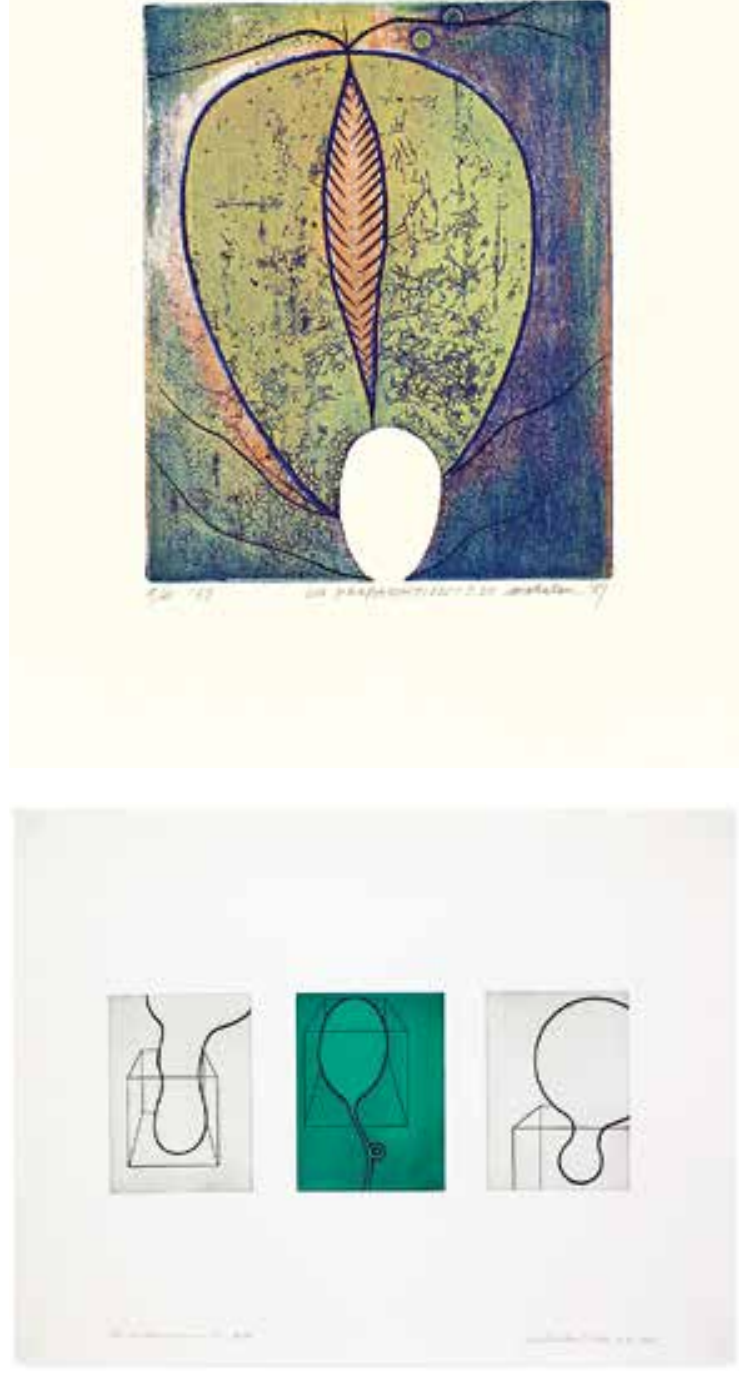

5. Takesada Matsutani, Propagation T-50, 1967, eau-forte et burin sur papier BFK, environ $65 \times 50 \mathrm{~cm}$, Paris, bibliothèque de l'Institut national d'histoire de l'art, inv. EM MATSUTANI 12.

6. Takesada Matsutani, La Propagation-M, 19671969, burin et eau-forte sur papier BFK, environ $50 \times 65 \mathrm{~cm}$, Paris, bibliothèque de l'Institut national d'histoire de l'art, inv. EM MATSUTANI 20.

- Takesada Matsutani. Oui, par exemple, dans À l'avenir (1968), j'ai tenté de représenter le plafond se reflétant dans la plaque de métal! Comme quand, dans ma jeunesse, je 

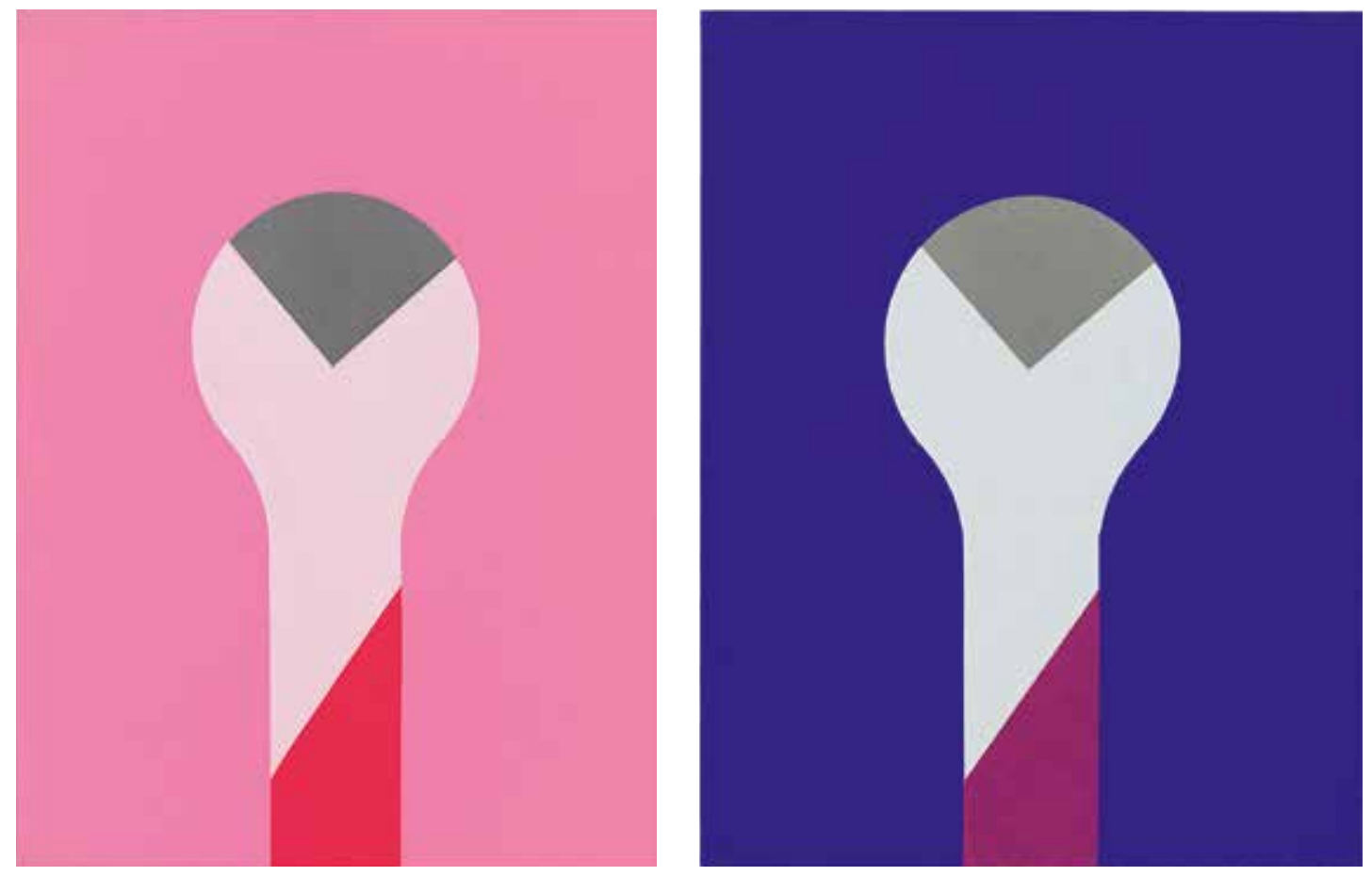

7a-c. Takesada Matsutani, Lamp-Pink [a], Lamp-Blue [b], Lamp-Silver [c], 1969, sérigraphies sur papier offset, environ

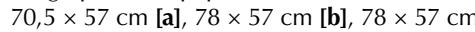
[c], Paris, bibliothèque de l'Institut national d'histoire de l'art, inv. EM MATSUTANI 26 [a], EM MATSUTANI 25 [b], EM MATSUTANI 27 [c] reproduisais ce que j'observais sur le plafond de ma chambre, quand je devais passer de longues périodes alité à cause de la tuberculose...

Comme je l'expliquais précédemment, ces gravures mélangent les formes organiques qui m'attirent depuis mes débuts et ma découverte de la perspective occidentale. Un peu comme Hokusai qui montre le mont Fuji en perspective lointaine à travers le tonneau que construit un personnage au premier plan. Je me demandais si Hokusai avait pu étudier la perspective européenne. En fait oui.

- Valérie Douniaux. Ces formes organiques dont vous parlez viennent d'une réelle observation n'est-ce pas?

- Takesada Matsutani. Oui, l'un de mes amis au Japon travaillait dans un laboratoire universitaire et m'a permis d'observer des cellules vivantes au microscope. Ça me fascinait. C'est aussi un symbole de vie, après ma victoire sur la maladie. D'où les titres de Développement, Propagation...

- Valérie Douniaux. Certaines de vos estampes, en particulier celles citées à l'instant, ont des titres clairement évocateurs, et intègrent parfois des éléments plus ou moins figuratifs dans la composition.

- Takesada Matsutani. C'est vrai. On oppose toujours figuration et abstraction. Mais par exemple la manière dont le cubisme montre divers points de vue d'un sujet me semble intéressante. 


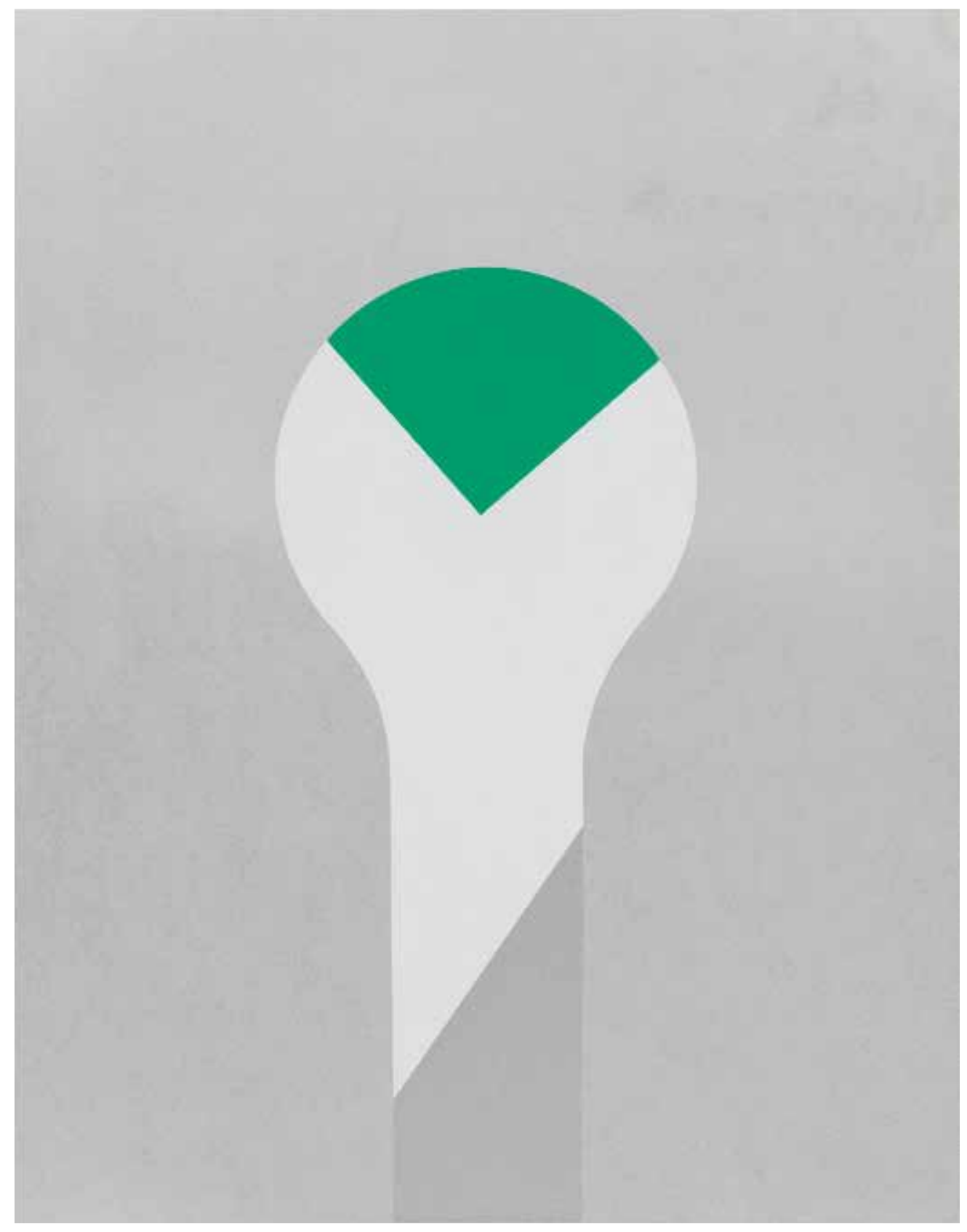

- Valérie Douniaux. Le travail de la forme et le noir et blanc prédominent dans vos premiers essais de gravure, mais la couleur ne disparaît pas totalement et elle revient même de manière éclatante quand vous passez à la sérigraphie. Elle est moins violente que dans vos peintures pré-Gutai et Gutai, dans lesquelles le rouge dominait. Il y a notamment un vert que vous semblez particulièrement affectionner puisqu'il revient souvent dans vos gravures et peintures des années 1970, et qu'on le retrouve encore dans vos œuvres les plus récentes. Il existe d'ailleurs pour certaines de vos estampes divers états avec des variations de tonalités de l'une à l'autre, voire des éditions très différentes, par exemple la série de sérigraphies intitulées Lamp (Lamp Pink, Lamp Blue, Lamp Silver [fig. 7a-c]...). 


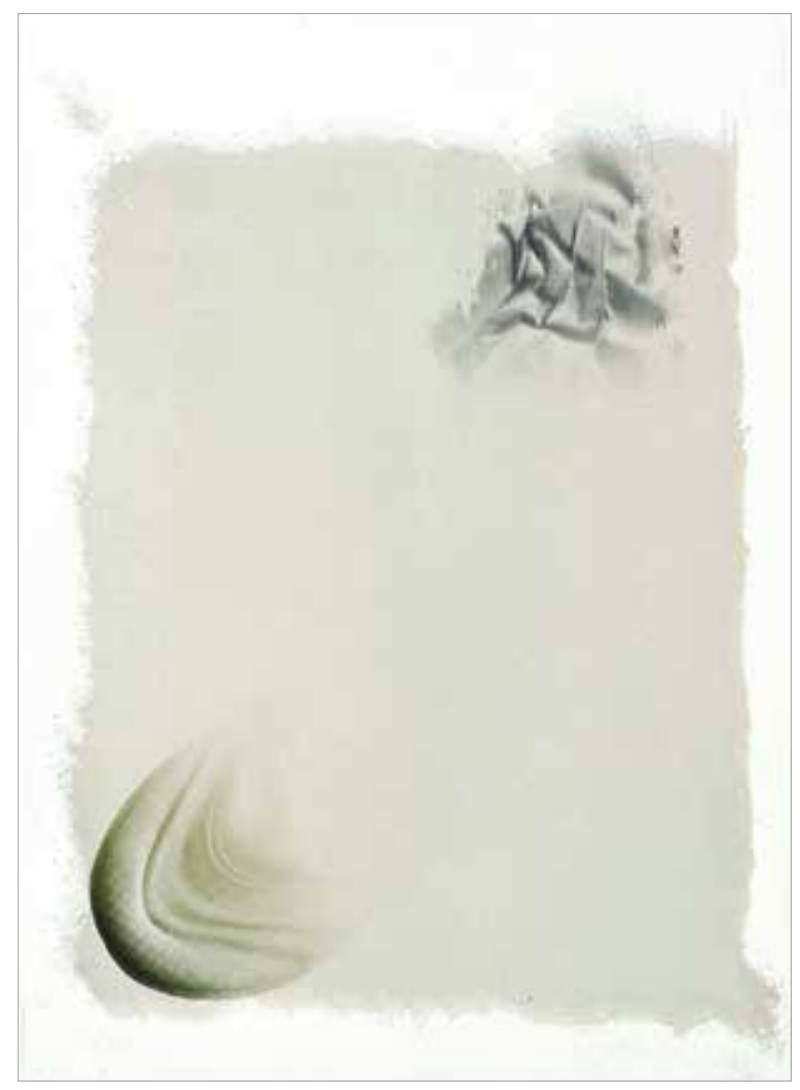

8. Takesada Matsutani, $F l y-K, 1974$, photosérigraphie sur papier, environ $75 \times 55 \mathrm{~cm}$, Paris, bibliothèque de l'Institut national d'histoire de l'art, inv. EM MATSUTANI 59.
- Takesada Matsutani. Oui, c'est vrai, j'affectionne ce vert, assez profond sans être agressif. Pour ce qui est des œuvres reproduites avec des variations, ce sont des recherches intuitives, j'aime changer constamment, c'est dans mon caractère et cela explique les changements dans ma carrière, par exemple mon retour à la couleur vive ces dernières années, ou mon départ de l'Atelier 17 pour rejoindre l'atelier de sérigraphie créé par ma femme Kate Van Houten et notre amie Lorna Taylor. J'ai rejoint leur atelier et j'ai appris la technique de la sérigraphie avec Kate. Nous nous partagions entre notre travail créatif et un travail de commande. J'étais enthousiasmé par la sérigraphie, la force colorée qu'elle permettait, la liberté qu'elle offrait par rapport à la gravure sur métal, les expérimentations qu'elle autorisait. Dans $F / y-K$ par exemple, j'ai utilisé du riz et du sable (fig. 8). Tout ceci se faisait spontanément, sur le moment, c'est toujours ainsi que je fonctionne.

- Valérie Douniaux. Oui, et malgré l'austérité méditative de votre travail au noir, vous n'avez jamais abandonné par ailleurs une certaine dimension ludique de l'acte créatif.

- Takesada Matsutani. Non, j'adore expérimenter, j'utilise tout ce que je trouve pour déclencher de nouvelles idées, inattendues. Et je laisse intervenir le hasard.

- Valérie Douniaux. Avez-vous gardé quelque chose de cette expérience de l'estampe et de la sérigraphie dans votre travail ultérieur?

- Takesada Matsutani. Tout d'abord, je n'ai pas totalement abandonné l'estampe, j'en fait encore ponctuellement, notamment pour des portfolios et des livres d'artistes. Le livre est un domaine que connaît très bien ma femme, qui a développé sa propre petite maison d'édition de livres d'artiste. Cependant, je n'ai pas de presse chez moi, je dois aller travailler dans d'autres ateliers. En plus, c'est très prenant, et je n'aime pas déléguer... Mais la gravure a été et reste importante pour moi. Elle est arrivée à un moment où je me redéfinissais comme oriental en Europe. Elle m'a permis de confirmer ce que j'avais pressenti à l'école en étudiant la calligraphie, la puissance et la profondeur du noir, l'importance de l'espace blanc. Elle m'a réappris aussi à envisager l'image à plat, et à revenir au travail de la main, après mes années dans Gutai à travailler les volumes à la colle vinylique. La gravure ne comporte pas la même part de hasard que mon travail avec la colle. Même si j'ai appris à diriger en partie ce hasard, le travail de gravure demande une idée plus précise au départ, et que l'idée précède l'image. 
- Valérie Douniaux. Vous citez fréquemment d'autres artistes, et encore actuellement vous montrez une vraie curiosité pour le travail des autres, et pour les jeunes artistes aussi, que vous encouragez.

- Takesada Matsutani. Oui, c'est important pour moi. J'ai beaucoup reçu, je suis heureux si je peux transmettre aussi. Je n'ai pas reçu une éducation académique poussée, ayant souvent manqué l'école à cause de la tuberculose. J'ai donc toujours eu le sentiment d'un manque à combler. J'observe et j'écoute beaucoup les autres artistes, et cela nourrit mon travail. Je pense que tous les artistes procèdent ainsi.

Quand je faisais partie de Gutai, nous allions tous voir les œuvres les uns des autres avant les expositions, nous discutions ensemble. À l'Atelier 17, c'était très animé, très international, avec peu de Français en fait, à part Jean-Claude Raynal et quelques autres. Je me sentais à l'aise au milieu de tous ces artistes,

9. Matsutani dans son atelier, posant à côté de Work-D (1971, acrylique sur toile découpée montée sur contreplaqué, $200 \times 240 \mathrm{~cm}$ ), archives Matsutani.

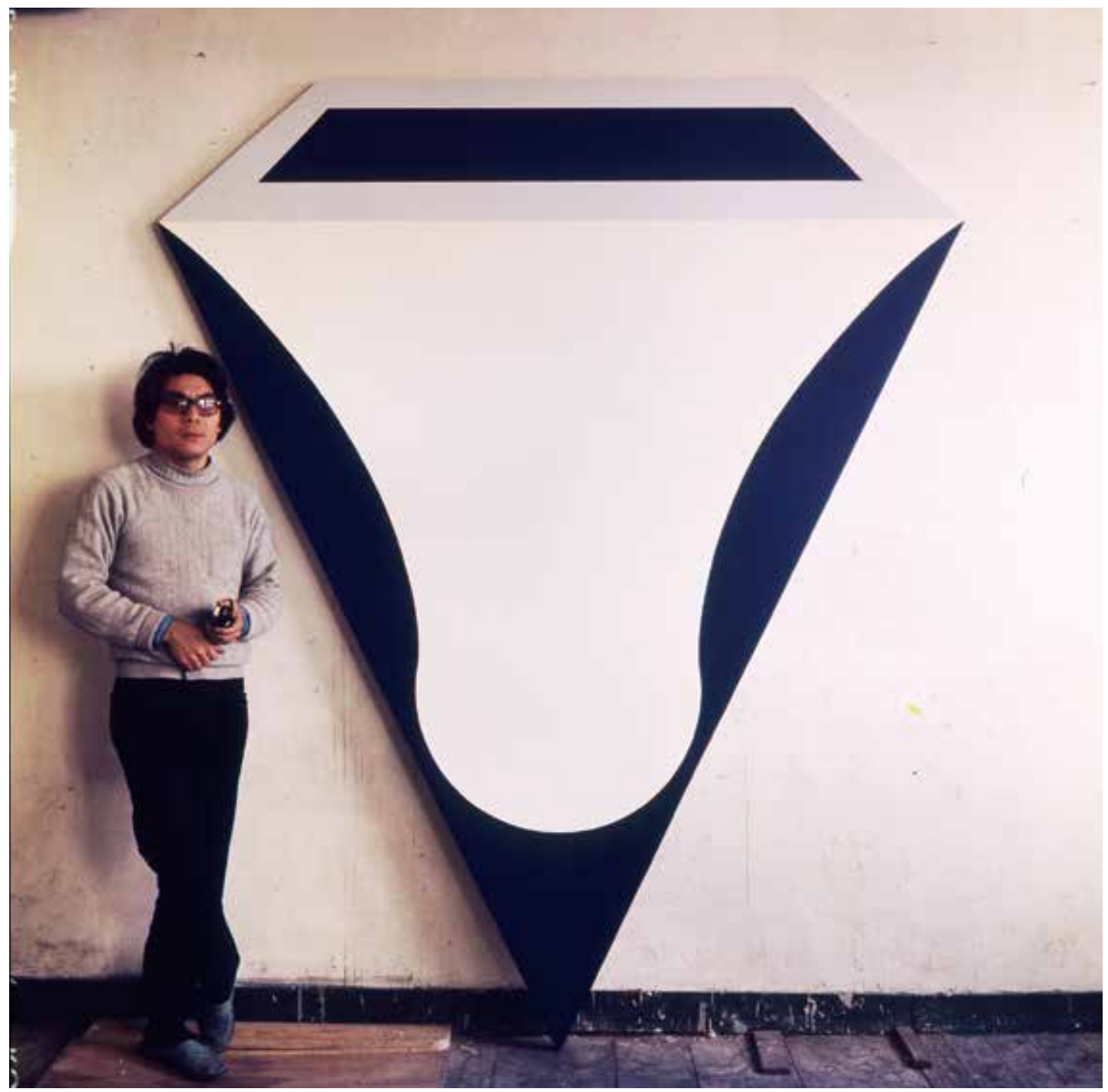


10. Takesada Matsutani, White Cloud, 1972 , huile sur toile, $162,2 \times 97 \times 2,54 \mathrm{~cm}$, Londres, collection particulière.

11. Takesada Matsutani, Object-S.U., 1978 photosérigraphie sur papier BFK, environ $55 \times 45 \mathrm{~cm}$, Paris, bibliothèque de l'Institut national $d$ 'histoire de l'art, inv. EM MATSUTANI 77. étrangers comme moi, et j'ai beaucoup appris sur les autres cultures. Les Français allaient plutôt chez Johnny Friedlaender, où l'enseignement était plus orthodoxe que chez Hayter, ou bien ils intégraient les Beaux-Arts et les Arts décoratifs.

Les observations de certains de mes amis m'ont aussi permis par la suite de trouver des réponses à certaines périodes importantes de ma carrière. Par exemple, c'est mon ami Horio Sadaharu (1939-2018) qui m'a suggéré d'utiliser de l'eau pour diluer l'encre, quand j'ai commencé à faire des installations et des performances au graphite. J'utilisais à l'origine de la térébenthine, mais l'odeur et l'agressivité de ce produit posaient problème. Et c'est un autre ami artiste, Shinjo Shigeo (1945-2014), qui m’a mis au défi justement de développer à grande échelle les bandes de graphite noir que j'ai commencé à faire au milieu des années 1970, au début sur des feuilles de papier de format standard.

- Valérie Douniaux. Justement, qu'est-ce qui a motivé ce passage de la gravure et des peintures Hard-Edge très colorées aux courants en noir et blanc au milieu des années 1970 ?

- Takesada Matsutani. La gravure prend trop de temps, le dynamisme de la création spontanée me manquait. J'effectuais des croquis dessinés à partir de mes motifs de prédilection, donc un travail direct de la main, mais il ne s'agissait généralement que de travaux préparatoires que je développais ensuite en peinture et en gravure, sans que l'une des techniques précède forcément l'autre selon un ordre bien défini. En tous cas, la gravure avait indéniablement pris le dessus sur toutes les autres formes d'expression. L'artiste coréen Chung Sang-Hwa (1932), en voyant les photosérigraphies

de mes anciennes œuvres Gutai en volume, m’a demandé pourquoi je m'escrimais à faire ainsi de la reproduction à plat plutôt que créer l'original en volume. Ça a été un déclencheur pour moi. J'étais tellement obsédé par la gravure et la sérigraphie que j'avais oublié la colle et le travail original, je m'étais laissé entraîner dans le processus de reproduction mécanique. En plus, je me suis trouvé presque obligé par les circonstances d'arrêter la sérigraphie : les produits utilisés à l'époque étaient très nocifs et mettaient notre santé en danger.

J'étais aussi gêné par les contraintes de l'image en petit format, imposées par le papier. Mon intérêt pour le Hard-Edge et surtout pour le travail d'Ellsworth Kelly (1923-2015), que j'avais pu découvrir dans des revues et lors d'expositions à Paris, m'avait déjà encouragé à retourner plus activement vers la peinture à la fin des années 1960 et au début des années 1970, à entreprendre des peintures très colorées, et lorsque j'ai enfin eu un atelier suffisamment grand, dans I'Impasse de la bonne graine, j'ai pu revenir aux grands formats (fig. 9). Cependant, j'ai rapidement ressenti aussi les limites de ce style, qui n'était pas à proprement parler le mien. 

Et puis, dans les années 1970, j'ai commencé à retourner régulièrement au Japon et j'ai tâché d'explorer I'histoire de l'art japonais comme je l'avais fait pour l'art occidental, j'ai visité les musées, les temples, les jardins. La lecture de l'Éloge de l'ombre de Tanizaki Junichirō ${ }^{2}$ a été déterminante. Je voulais accéder à une nouvelle étape de mon travail. J'avais du temps libre mais peu de moyens. Je suis donc revenu aux fondamentaux, le papier blanc et le graphite noir. Et j'ai commencé à recouvrir les feuilles de papier blanc avec des traits de crayon noir. Les strates de noir créaient des jeux d'ombre riches comme l'ombre qu'évoque Tanizaki. Je m'intéressais aux concepts esthétiques japonais, notamment celui de yohaku, la surface laissée blanche, aussi importante dans l'art japonais ou chinois que la surface peinte, avec laquelle elle établit une relation, un équilibre. Quelque chose que j'avais ressenti en gravure aussi. J'ai été très impressionné par une exposition, que j'ai vue au Japon, des chefs-d'œuvre de Tōhaku Hasegawa (1539-1610), ses pins dans la brume (Musée national de Tōkyō, inv. A10471), qui sont une des plus célèbres manifestations du yohaku. Tout est suggéré par les seuls noir et blanc, l'encre et le papier. Le blanc n'est pas vide, il exprime l'espace, l'atmosphère, l'esprit du lieu et il établit une relation avec le noir. L'un existe grâce à l'autre. Devant les œuvres de Hasegawa, j'ai ressenti une grande émotion et j'ai pressenti ce à quoi je souhaitais parvenir dans mon propre travail.

- Valérie Douniaux. Vous parlez de l'importance du blanc. On vous associe le plus souvent au noir mais vous avez créé aussi un certain nombre d'œuvres blanches, des sérigraphies et peintures dans lesquelles les formes blanches fantomatiques se fondent avec la surface blanche elle aussi, se distinguant juste par de

12. Takesada Matsutani, Germination 90-7-14, 1990, colle vinylique, crayon graphite, acrylique,

papier japonais marouflé sur toile, $162,1 \times 130,3 \mathrm{~cm}$.

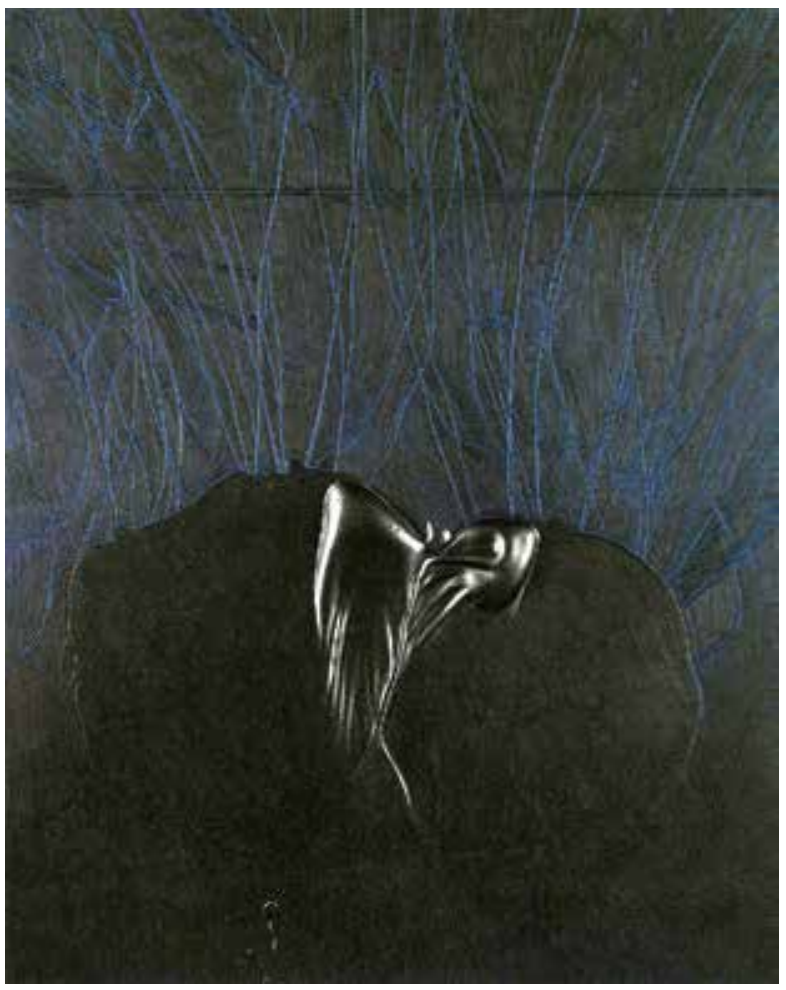
subtiles nuances de matière et de dégradés.

- Takesada Matsutani. Oui, le blanc m'attire beaucoup et j'ai fait à cette période de transition de nombreuses œuvres blanches (fig. 10), dont la plupart ont malheureusement disparu. En fait, j'aime la transparence, le suggéré. La colle à bois devient d'ailleurs transparente en gonflant, à travers elle on voit la toile.

- Valérie Douniaux. Il existe également un certain nombre de sérigraphies (conçues à partir de photographies de vos œuvres Gutai en volume), par exemple Object S.U. (fig. 11), dans lesquelles les formes semblent s'effacer, où le blanc ou les couleurs pâles semblent se dissoudre en dégradés.

- Takesada Matsutani. C'est directement inspiré des ukiyo-e. Ou des nuages qui suggèrent l'espace dans la peinture traditionnelle japonaise.

- Valérie Douniaux. Mais finalement vous êtes allé vers l'opposé et vous êtes consacré au noir. 
- Takesada Matsutani. Je voulais explorer la richesse de l'ombre. J'aimais aussi le fait que le noir, le trait de crayon graphite, garde la trace de la main. C'est toujours une grande problématique, surtout actuellement, alors que les artistes utilisent de plus en plus les nouvelles technologies. II me semble important de garder cette dimension manuelle, ce côté vivant du travail de l'artiste.

- Valérie Douniaux. La colle à bois, qui était votre matériau privilégié de l'époque Gutai, a fait son retour aussi dans votre travail, et vous avez commencé à associer les deux, les bandes de graphite noir et les volumes de colle.

- Takesada Matsutani. J'avais envie de réutiliser la colle. Et celle-ci, associée au noir, permettait de jouer encore plus avec l'ombre, en créant des volumes, des replis (fig. 12)...

- Valérie Douniaux. Et cela fait surgir dans la planéité des rouleaux noirs la dimension organique qui vous est si chère depuis vos débuts.

- Takesada Matsutani. Oui, au final, j'espère que je suis resté fidèle à la devise de Gutai, de chercher constamment à faire « ce qui n’a jamais été fait » et au principe Gutai de dialoguer avec la matière, de laisser intervenir le hasard, le geste spontané. 


\section{NOTES}

1. Takesada Matsutani : estampes, 1967-1977, Collections de l'Institut national d'histoire de l'art, Toulouse, Les Abattoirs, du 28 février au 31 mai 2020.

2. Tanizaki Junichirō, In'ei raisan, 1933. Traduit en 1977 par René Sieffert, paru aux PUF, réédité en 2011 chez Verdier. Voir la nouvelle traduction, sous le titre Louange de l'ombre, par Ryōko Sekiguchi et Patrick Honnoré, Arles, Éditions Philippe Picquier, 2017. 